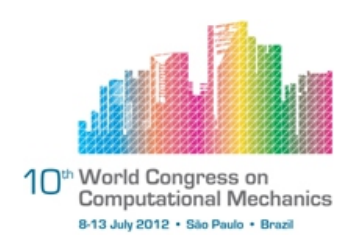

\title{
Bi-directional Evolutionary Topology Optimization for Multiphysics Problems with Frequency Response Minimization
}

\author{
W. M. Vicente ${ }^{1}$, R. Picelli ${ }^{1}$, R. Pavanello ${ }^{1}$ \\ ${ }^{1}$ Department of Computational Mechanics, Faculty of Mechanical Engineering at the State \\ University of Campinas (vicente@fem.unicamp.br)
}

\begin{abstract}
This work presents a topology optimization for frequency responses in multiphysics problems involving fluid-structure interaction. A mixed formulation $(u / p)$ is used, in which the pressure and displacement are governed by Helmholtz equation and the elasticity equation, respectively. The optimization method used in this work is the Bi-directional Evolutionary Structural Optimization (BESO), which consists in a successive elimination and replacement of elements in the design domain. The feasible space of solution is defined initially and through a sensitivity analysis of the frequency response functions the evolutionary algorithm remove or add solid elements. The sensitivity analysis is described for the dynamic problems and the sensitivity numbers are evaluated for several conditions. The formulation implemented in FORTRAN aims to determine the optimum topology in order to minimize the displacement and/or pressure in specific parts of the system for a certain range of frequency excitation. A number of final topologies for vibroacoustic problems are shown, as well as their intermediary topologies and evolutionary history. The results show that this methodology can be applied to this type of problem with good efficiency.
\end{abstract}

Keywords: Frequency Response Functions, Topology Optimization, BESO Method.

\section{INTRODUCTION}

In order to improve the vibration or pressure characteristics of a system, topology optimization might be applied [1]. The basic idea is to find an optimal distribution of material in a structural design domain, considering an objective function. In this work, we investigated how the vibration or pressure characteristics of given examples can be improved on the basis of the structural frequency response function (FRF) with topology optimization.

Between researches considering dynamic responses, we can cite the work of Diaz and Kikuchi [2], where they applied topology optimization by considering the eigenvalues of a structure for optimal plate reinforcement using the homogenization method. Ma et al. [3] extended the same method to problems of vibrating structures. The topology optimization considering dynamic responses was also investigated with the classical material interpolation method 'SIMP' (Solid Isotropic Material with Penalization) [1]. Many efforts have been made to develop and optimize dynamic systems. Frequency optimization is of great importance in many engineering fields and dynamic responses must be took in account in a wide range 
of problems. One of these problems is the class of multiphysics systems. The applications of topology optimization methods have also been extended to multiphysics problems [4-6]. Although these procedures have reached a satisfactory level of maturity, there are still many topology optimization features open to research or less-than-satisfactorily resolved issues [6]. Commercial FEM packages often contain solvers for multiphysics problems, however they do not enable optimization. A particular class of multiphysics problems involves fluid-structure interaction (FSI), which is the main topic of this work.

Among the current optimization methods, the Bi-directional Evolutionary Structural Optimization (BESO) was chosen in this work for FSI problems analysis, since the method is almost not explored in this area. The technique so called Evolutionary Structural Optimization (ESO) was first introduced in the 90's with Xie and Steven [7]. With the finite element analysis, the ESO method was initially proposed as a gradual removal of inefficient material from the design domain until the remaining structure converges to the optimum topology. Material elimination is done after a sensitivity analysis. However, it is questionable that eliminated elements can never return to the design domain, since the sensitivity analysis is carried out only for the solid elements in the structure. Thus, a later development of this method was called Bi-directional ESO (BESO), in which elements are also added in void positions near to the elements with the highest sensitivity numbers [8]. In this case, the sensitivity number is a local index and represent the sensitivity of each element with respect to the objective function. Studies with the BESO method have recently been published presenting convergent and mesh independent solutions for stiffness maximization [9], for natural frequency maxi-

mization [10] and others. Critical analysis of ESO-based methods are found in [11], while a later review about the ESO/BESO methods is found in [12] and a book is also available [13]. In multiphysics problems, the ESO/BESO methods lack of methodologies and publications, especially in fluid-structure coupled systems.

Herewith it is proposed to combine the evolutionary topology optimization techniques with multiphysics problems involving fluid-structure interaction. This work focuses on the mean pressure minimization in coupled systems. Frequency response optimization is of great importance in many engineering fields like acoustics systems and fluid dynamics. The possibility of removing and adding material systematically with the evolutionary method might be a helpful procedure to explicitly define the fluid-structure interfaces. However, only cases with immovable interfaces will be considered in this paper. The paper is organized as follows: Section 2 presents the governing equations and the finite element model for the fluid-structure interaction coupled system. In Section 3, the topology optimization problem for pressure minimization is formulated and the sensitivity number is presented. Section 4 shows two examples of the optimization methodology, BESO.

\section{FLUID-STRUCTURE INTERACTION: GOVERNING EQUATIONS AND FINITE ELEMENT MODEL}

Herein, the analyzed systems are limited to a flexible structure in contact with an enclosed acoustic fluid, where the responses of the structure are significantly affected by the fluid. For this fluid-structure system, the structure can be described by the differential equation of motion for a continuum body assuming small deformations and the fluid by the acoustic 
wave equation. For the standard approach, the governing equations for the fluid and structural domains as well as the coupling boundary conditions are defined as follows.

\subsection{Helmholtz equation}

In this paper, the fluid is considered inviscid, irrotational and only under small translations conditions. The governing equation for the pressure in a nonhomogeneous acoustic medium is described by the Helmholtz equation

$$
\nabla^{2} p_{f}+\frac{\omega^{2}}{c_{f}^{2}} \frac{\partial^{2} p_{f}}{\partial t^{2}}=0 \quad\left(k=\frac{\omega}{c_{f}}\right) \quad \Omega_{f}
$$

where $p_{f}$ is the pressure in the analysis domain $\Omega_{f}, c_{f}$ is the local speed of sound, $\omega$ is the angular frequency and $k$ the wave number. The pressure field is obtained by solving the Helmholtz equation imposing proper boundary conditions. In this paper, it is considered the following boundary conditions:

$$
\begin{gathered}
p_{f}=p_{0} \\
\mathbf{n} \nabla p_{f}=0
\end{gathered}
$$

representing the pressure boundary condition (Eq. 2) and the hard wall condition (Eq. 3), where $p_{0}$ is the pressure input and $\mathbf{n}$ is the outward unit normal to the fluid.

\subsection{Linear elasticity}

The linear structural analysis can be described by the elasticity equation

$$
\tilde{\nabla}^{T} \sigma_{s}+\mathbf{b}_{s}=\rho_{s} \frac{\partial^{2} \mathbf{u}_{s}}{\partial t^{2}} \quad \Omega_{s}
$$

where $\sigma_{s}$ is the stress tensor, $\mathbf{b}_{s}$ are the body forces and $\mathbf{u}_{s}$ is the displacements field. Boundary conditions are applied as follows:

$$
\begin{array}{ll}
\mathbf{n}_{s} \sigma=\mathbf{f}^{S_{b}} & S_{b} \\
\mathbf{u}_{s}=\mathbf{u}_{s}^{S_{u}} & S_{u}
\end{array}
$$

representing the Neumann (Eq. 5) and Dirichlet (Eq. 6) boundary conditions, where $\mathbf{f}^{S_{b}}$ is the surface traction on $S_{b}, \mathbf{u}_{s}^{S_{u}}$ is the prescribed displacements on $S_{u}$ and $\mathbf{n}_{s}$ is the outward unit normal to the solid medium.

\subsection{The coupled fluid-structure system}

At the interface $\partial \Omega_{s f}$ between the structural and fluid domains, the fluid and the structure move together in the normal direction of the boundary. The normal vector $\mathbf{n}=\mathbf{n}_{f}=-\mathbf{n}_{s}$ can be used in order to obtain the displacement boundary condition 


$$
\left.\mathbf{u}_{s} \mathbf{n}\right|_{\partial \Omega_{s f}}=\left.\mathbf{u}_{f} \mathbf{n}\right|_{\partial \Omega_{s f}}
$$

and the continuity in pressure

$$
\left.\sigma_{s}\right|_{n}=-p
$$

With relations derived from the governing equations and the previous coupling conditions, the interface forces may be obtained. The force acting on the structure provided by the fluid pressure is

$$
\mathbf{f}_{f}=\int_{\Omega_{s f}} \mathbf{N}_{s}^{T} \mathbf{n} \mathbf{N}_{f} d s \mathbf{p}_{\mathbf{f}}
$$

and the force acting on the fluid domain can be expressed in structural acceleration

$$
\mathbf{f}_{s}=-\rho_{f} \int_{\Omega_{s f}} \mathbf{N}_{f}^{T} \mathbf{n} \mathbf{N}_{s} d s \ddot{\mathbf{u}}_{\mathbf{s}}
$$

where $\rho_{f}$ is the static density of the fluid and $\mathbf{N}$ contains the finite element shape functions for the interface.

The introduction of a spatial coupling matrix

$$
\mathbf{L}_{s f}=\int_{\Omega_{s f}} \mathbf{N}_{s}^{T} \mathbf{n} \mathbf{N}_{f} d s
$$

allows the coupling force to be written as

$$
\mathbf{f}_{f}=\mathbf{L}_{s f} \mathbf{p}_{f}
$$

and

$$
\mathbf{f}_{s}=-\rho_{f} \mathbf{L}_{s f}^{T} \ddot{\mathbf{u}}_{s}
$$

Thus, the fluid-structure problem can then be described by an unsymmetrical system of equations

$$
\begin{gathered}
{\left[\begin{array}{cc}
\mathbf{M}_{s} & \mathbf{0} \\
\rho_{f} \mathbf{L}_{s f}^{T} & \mathbf{M}_{f}
\end{array}\right]\left[\begin{array}{c}
\ddot{\mathbf{u}}_{s} \\
\ddot{\mathbf{p}}_{f}
\end{array}\right]+\left[\begin{array}{cc}
\mathbf{K}_{s} & -\mathbf{L}_{s f} \\
\mathbf{0} & \mathbf{K}_{f}
\end{array}\right]\left[\begin{array}{l}
\mathbf{u}_{s} \\
\mathbf{p}_{f}
\end{array}\right]=\left[\begin{array}{l}
\mathbf{f}_{s} \\
\mathbf{f}_{f}
\end{array}\right]} \\
{\left[\mathbf{M}_{f s}\right]\left[\ddot{\mathbf{u}}_{f s}\right]+\left[\mathbf{K}_{f s}\right]\left[\mathbf{u}_{f s}\right]=\left[\mathbf{f}_{f s}\right]}
\end{gathered}
$$

where $\mathbf{f}_{s}$ and $\mathbf{f}_{f}$ are the load vectors for both domains.

In the multiphysics coupling analysis, the fluid analysis provides pressure loads to the structural analysis, and the structural analysis provides accelerations to the fluid analysis. These finite element model has been used to calculate responses of acoustic-structure interaction problems. For details and derivations of the coupling integrals as well as the governing equations, see [14-15]. 


\section{BI-DIRECTIONAL EVOLUTIONARY STRUCTURAL OPTIMIZATION}

\subsection{Problem Statement}

Considering volume constraint, the optimization problem of frequency response minimization can be stated as:

$$
\begin{array}{cc}
\text { Minimize } & \left|\mathbf{u}_{f s}\right| \\
\text { Subject to: } & V_{f}-\sum_{i=1}^{n} V_{i} x_{i}=0 \\
& x_{i}=x_{\text {min }} \text { or } 1
\end{array}
$$

where $\mathbf{u}_{f s}$ is the frequency response, $V_{i}$ is the volume of an individual element, $V_{f}$ the prescribed final structural volume and $n$ is the total number of elements in the system. The binary design variable $x_{i}$ declares the presence of a completely solid element (1) or the density of a void element with a small value of $x_{\min }$ (e.g. $10^{-4}$ ).

\subsection{Vibroacoustic sensitivity}

The vibroacoustic sensitivity analysis involves the evaluation of the fluid-structure coupled system response under a structural change. The objective here is to evaluate the sensitivity of the vibroacoustic response $\left|\mathbf{u}_{f s}\right|$ with respect to the variables $x_{i}$.

The residual $R_{f s}$ of the governing equation of the coupled dynamic problem is given by:

$$
\begin{aligned}
R_{f s}= & \left(\mathbf{K}_{f s}-\omega^{2} \mathbf{M}_{f s}\right)\left|\mathbf{u}_{f s}\right|-\left|\mathbf{f}_{f s}\right| \\
& R_{f s}=\mathbf{Z}\left|\mathbf{u}_{f s}\right|-\left|\mathbf{f}_{f s}\right|
\end{aligned}
$$

With the analytical derivatives of equation 16 and analyzing the partial derivatives of $R_{f s}$ with respect to $\left|\mathbf{u}_{f s}\right|$ and $x_{i}$, the sensitivity of the coupled system response $\left|\mathbf{u}_{f s}\right|$ is:

$$
\frac{d\left|\mathbf{u}_{f s}\right|}{d x_{i}}=-\mathbf{Z}^{-1} \frac{\partial \mathbf{Z}}{\partial x_{i}}\left|\mathbf{u}_{f s}\right|
$$

It was observed that the sensitivity from Equation 17 is equivalent to the following expression:

$$
\frac{d\left|\mathbf{u}_{f s}\right|}{d x_{i}}=\mathbf{u}_{i j} \mathbf{Z} \mathbf{u}_{i}=\alpha_{i j}
$$

where $\mathbf{u}_{i j}$ is the solution of the dynamic equilibrium equation $\mathbf{Z} \mathbf{u}_{j}=\mathbf{F}_{j}$ with an unit load vector $F_{j}$ at the $j$-th objective node. $\mathbf{u}_{i}$ is the displacement vector containing the entries of $\mathbf{u}_{f s}$, which is related to the $i$-th element. This sensitivity number $\alpha_{i j}$ is equivalent to that proposed by [16] for stiffness maximization with displacement constraints. It indicates the change of the specified displacement or pressure component $\mathbf{u}_{j}$ due to the removal of the $i$ th element. This number was initially proposed for static cases. Here, we combine it with the interpolation scheme proposed by [10] for the BESO method. In the proposed scheme, material is interpolated and not removed completely from the domain. It implies that the interpolation depends on $x_{\min }$ and an exponent penalty factor $p$, which indicate how soft is 
the void-condition elements. Thus, the sensitivity numbers proposed for frequency response minimization are the following:

$$
\begin{aligned}
\alpha_{i}=\frac{1}{p} \frac{d \mathbf{u}_{f s}}{d x_{i}} & =\frac{1}{2 \omega} \mathbf{u}_{j}^{T}\left(\frac{1-x_{\min }}{1-x_{\min }^{p}} \mathbf{K}_{i}^{1}-\frac{\omega^{2}}{p} \mathbf{M}_{i}^{1}\right) \mathbf{u}_{i} & & x_{i}=1 \\
& =\frac{1}{2 \omega} \mathbf{u}_{j}^{T}\left(\frac{x_{\min }^{-1}-x_{\min }^{p}}{1-x_{\min }^{p}} \mathbf{K}_{i}^{1}-\frac{\omega^{2}}{p} \mathbf{M}_{i}^{1}\right) \mathbf{u}_{i} & & x_{i}=x_{\min }
\end{aligned}
$$

or when $x_{\min }$ tends to 0

$$
\begin{aligned}
\alpha_{i}=\frac{1}{p} \frac{d \mathbf{u}_{f s}}{d x_{i}} & =\frac{1}{2 \omega} \mathbf{u}_{j}^{T}\left(\mathbf{K}_{i}^{1}-\frac{\omega^{2}}{p} \mathbf{M}_{i}^{1}\right) \mathbf{u}_{i} & & x_{i}=1 \\
& =-\frac{\omega}{p} \mathbf{u}_{j}^{T} \mathbf{M}_{i}^{1} \mathbf{u}_{i} & & x_{i}=x_{m i n}
\end{aligned}
$$

\subsection{Evolutionary Procedures for pressure minimization}

The BESO method allows material to be removed and added trough the initial domain covered by a fine finite element mesh until an optimum solution is found. A sensitivity analysis of the problem is needed for the adopted criterion. For dynamic problems, considering displacement and/or pressure minimization in cases of fluid-structure interaction, when a solid element is removed from the structure, the change on the frequency response is evaluated by the dynamic compliance. This change is defined as the elemental sensitivity number from Equation (20). In the BESO process, the elements are organized according to their values of the sensitivity number, from the highest to the lowest. For pressure minimization is reasonable to remove elements with the lowest $\alpha$.

A filter scheme is applied over the mesh in order to smooth the sensitivity numbers distribution. This filter scheme is similar to the mesh-independency filter used in ([17]. This procedure consists, first of all, in averaging the elemental sensitivity number to the mesh nodes, according to the nodal connectivity, as follows:

$$
\alpha_{j}^{n}=\frac{\sum_{i=1}^{M} V_{i} \alpha_{i}}{\sum_{i=1}^{M} V_{i}}
$$

where $M$ is the total number of elements connected to the node $j$.

The above nodal sensitivity numbers will then be converted into smoothed elemental sensitivity numbers by projecting a sub-domain $\Omega$ with length scale $r_{\min }$ and center in the $i$ th element. All the nodes inside $\Omega$ will have their nodal sensitivity numbers averaged back to the elemental level, now as a smoothed projection. Thus, the sensitivity numbers of void elements are automatically obtained.

To stabilize the evolutionary process, an average of the sensitivity number with its historical information is carried out as:

$$
\alpha_{i}=\frac{\alpha_{i}^{k}+\alpha_{i}^{k-1}}{2}
$$

where $k$ is the current iteration number. Thus, the updated sensitivity number includes the whole history of the sensitivity information in the previous iterations.

The removal/addition of elements is carried out by the target volume of the next iteration $\left(V_{k+1}\right)$, defined as: 


$$
V_{k+1}=V_{k}(1 \pm E R)
$$

where $E R$ is the evolutionary ratio. Once the volume constraint is satisfied, the volume of the structure will be kept constant for the remaining iterations as:

$$
V_{k+1}=V_{f}
$$

With the target volume evaluated, the threshold sensitivity numbers ( $\alpha_{d e l}^{a t h}$ and $\left.\alpha_{a d d}^{a t h}\right)$ are determined. For solid element (1), it will be removed (switched to 0 ) if:

$$
\alpha_{i} \leq \alpha_{d e l}^{a t h}
$$

For void elements (0), it will be added (switched to 0 ) if:

$$
\alpha_{i} \leq \alpha_{a d d}^{a t h}
$$

An admission volume ratio $(A R)$ is introduced to ensure that not too many elements are added in a single iteration. $A R$ is defined as the number of added elements divided by the total number of elements in the design domain. If $A R$ is bigger than a prescribed value $A R_{\text {max }}$, then $\alpha_{d e l}^{a t h}$ and $\alpha_{\text {add }}^{a t h}$ need to be recalculated. More details are found in [9].

Besides the volume constraint, a convergence criterion is introduced to stop the algorithm. The cycle of finite element analysis and element removal and addition continues until the objective volume $\left(V_{f}\right)$ is reached and the following convergence criterion defined in the variation of the objective function is satisfied:

$$
\text { error }=\frac{\left|\sum_{i=1}^{N} \mathbf{u}_{k-i+1}-\sum_{i=1}^{N} \mathbf{u}_{k-N-i+1}\right|}{\sum_{i=1}^{N} \mathbf{u}_{k-i+1}} \leq \tau
$$

where $k$ is the current iteration number, $\tau$ is an allowable convergence error and $N$ is integral number, herewith selected as 5, which means a stable compliance at least in successive $2 * N$ iterations, i.e., 10 iterations in this case.

In short, the evolutionary iteration procedure of the present BESO method is given as follow:

1. Discretize the design domain using a fine finite element mesh and declare the initial values of the properties ( 0 or 1$)$ of the elements to construct an initial design.

2. Carry out the finite element analysis and calculate the elemental sensitivity numbers, according to Equation (20). Keep the sensitivity number for the next iteration.

3. Determine the target volume of the next iteration, according to Equation (23).

4. Add and remove elements according to the procedure described previously.

5. Repeat steps 2 to 5 until the volume constraint $\left(V_{f}\right)$ is reached and the convergence criterion (27) is satisfied. 
As a consequence of the removing material the natural frequencies of the system are changed. The natural frequencies closer to the excitation frequency point in the FRF curve can increase or decrease depending on which side they are relatively to the excitation frequency point. This occurs as a consequence of the minimization process which tends to make the point of optimization to go down in the FRF curve.

\section{NUMERICAL RESULTS}

In this section, two examples considering FSI are solved using the BESO method. The meshes were generated in ANSYS, the optimizer code was implemented in FORTRAN and the topologies were plotted in MATLAB. All the steps were automatically aggregated. In the first example, the intermediary topologies are presented to show the objective function and pressure distribution in several steps of the optimization. The second example presents the optimization process for six different excitation frequencies.

\subsection{Example 1}

This first example shows the FSI problem, where two fluid cavities are separated by a structure partition, Fig. ??. The objective is to minimize the mean pressure in the two output points for a specific excitation frequency. Hard-wall condition and a prescribed pressure were imposed to the fluid domain and the structure domain has clamped ends. The incoming wave amplitude of the excitation is $p_{\text {in }}=1 \mathrm{~Pa}$, in the left fluid domain. The excitation frequency for optimization is $5 \mathrm{~Hz}$. The structure domain has length of $1 \mathrm{~mm}$, height $10 \mathrm{~mm}$ and represents the design domain. For the optimization procedure the design domain is divided using a regular mesh of $64 \times 320$ four-node quadrilateral elements, totaling 20480 elements.

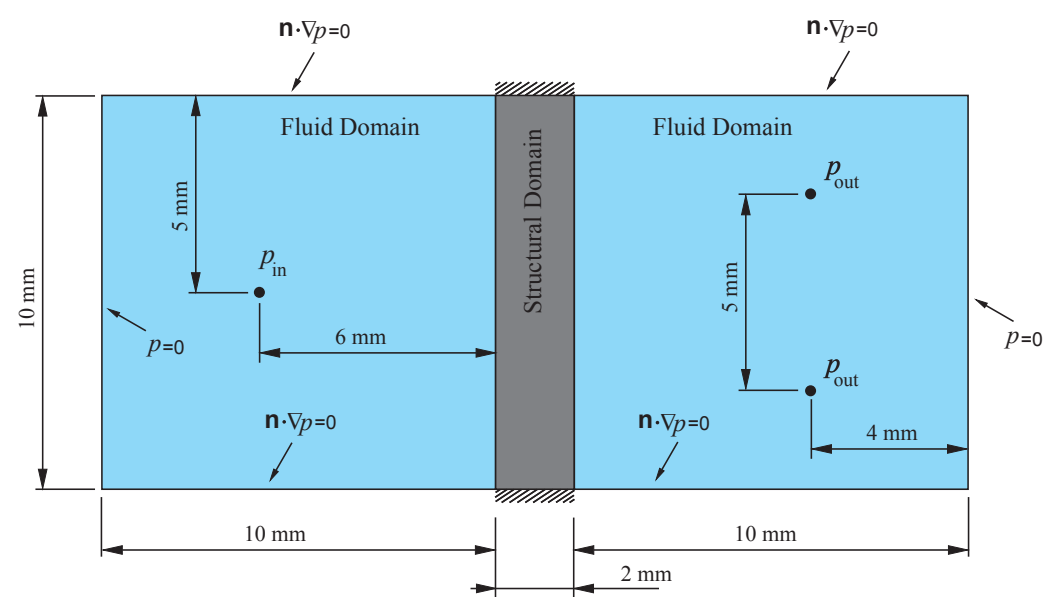

Figure 1. Model considered for optimization.

The parameters for the mesh in the finite elements analysis and the materials properties for fluid and structure domain are presented in Tab. 1. All constants related to the optimization process (BESO) are also listed in Tab. 1.

Figure 2 shows the FRF for the initial full design domain and the FRF after the optimization procedure for the mean pressure in the output point in second fluid domain. 
Table 1. Parameters considered for the numerical modelling.

\begin{tabular}{|c|c|c|}
\hline Variable & Description & Value \\
\hline \multicolumn{3}{|c|}{ Mesh } \\
\hline nnos & Number of nodes & 60465 \\
\hline nele & Number of elements & 60160 \\
\hline \multicolumn{3}{|c|}{ Structure } \\
\hline$E$ & Young's Modulus & $100 \mathrm{GPa}$ \\
\hline$\nu$ & Poisson coefficient & 0,3 \\
\hline$\rho_{s}$ & Density & $100 \mathrm{~kg} / \mathrm{m}^{3}$ \\
\hline \multicolumn{3}{|c|}{ Fluid } \\
\hline$\rho_{f}$ & Density & $1.2 \mathrm{~kg} / \mathrm{m}^{3}$ \\
\hline$c$ & Sound velocity & $343 \mathrm{~m} / \mathrm{s}$ \\
\hline \multicolumn{3}{|c|}{ BESO } \\
\hline$V_{i}$ & Initial volume fraction & 1,0 \\
\hline$V_{f}$ & Final volume fraction & 0,9 \\
\hline$E R$ & Evolutionary ratio & 0,02 \\
\hline$A R_{\max }$ & Maximum admission ratio & 0,02 \\
\hline$r_{\min }$ & Filter radius & $0,02 \mathrm{~mm}$ \\
\hline$\tau$ & Convergence tolerance & 0,001 \\
\hline$N$ & Integral number & 5 \\
\hline$p$ & Penalty number & 1.5 \\
\hline
\end{tabular}

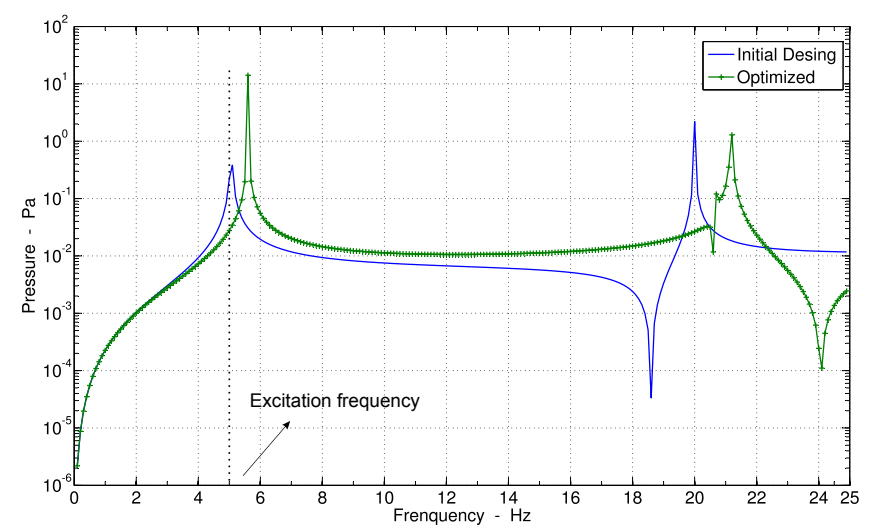

Figure 2. Frequency response for example 1.

The history of the mean pressure in the output points are shown in Fig. 3. This figure shows that the optimization process starts from a full design domain (Structural Domain) and regularly removes material in a rate of $0.2 \%$ until it reaches the final volume. After 50 iterations the final volume is reached and the process is finalized when the convergence criterion is obtained, in this case in the 58th iteration. The total pressure reduction after the optimization process can be found in Tab. 2.

The intermediaries and final topologies are showed in Fig. 4. The pressure distribution in both cavities and the shape of vibration of the structure domain can also be seen in Fig. 4 . The displacement of the structure is plotted $100 \times$ bigger for a better visualization of the shape of vibration for this excitation frequency. 


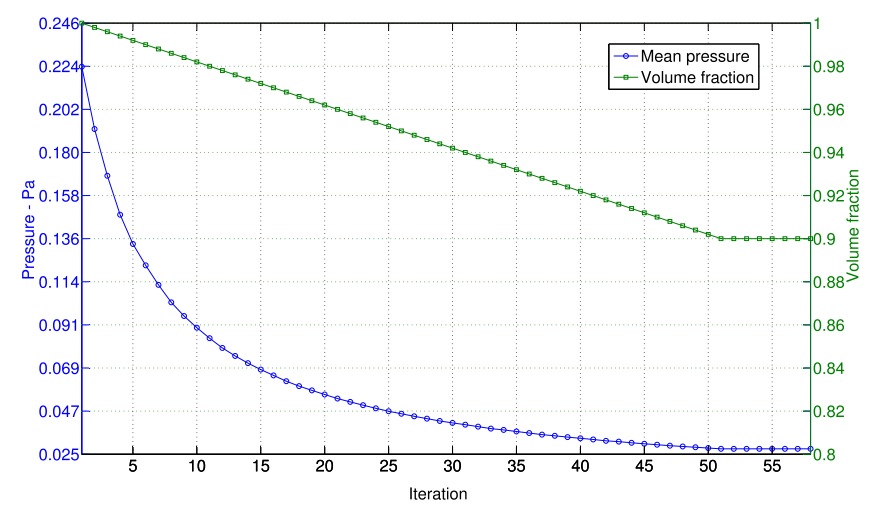

Figure 3. Mean pressure history for example 1.

Table 2. Mean pressure reduction at output points.

\begin{tabular}{|c|c|}
\hline Excitation Frequencies $(\mathrm{Hz})$ & 5.0 \\
\hline Initial pressure $(\mathrm{Pa})$ & $2.237 \mathrm{E}-01$ \\
\hline Final pressure $(\mathrm{Pa})$ & $0.278 \mathrm{E}-01$ \\
\hline Reduction & $87.58 \%$ \\
\hline
\end{tabular}

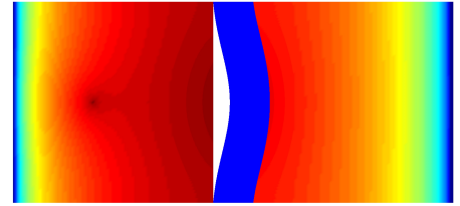

a)

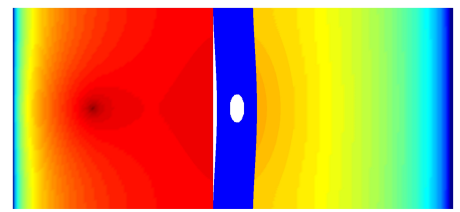

c)

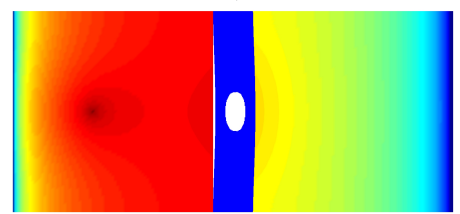

e)

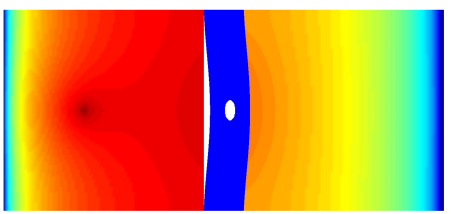

b)

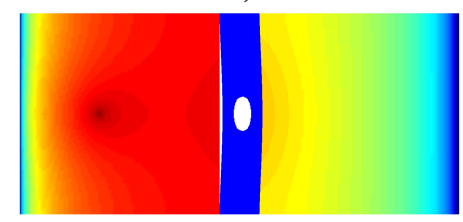

d)

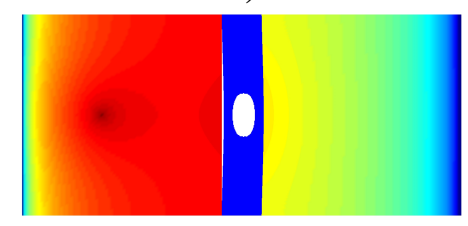

f)

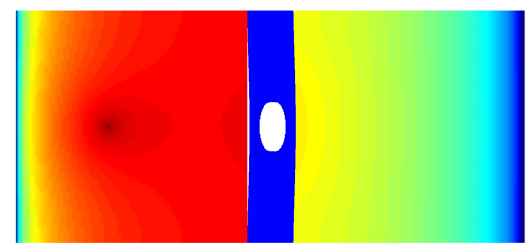

g)

Figure 4. Evolutionary topologies for Example 1 with material volume of: a) $100 \%$ - Design Domain; b) $98 \%$; c) $96 \%$; d) $94 \%$; e) $92 \%$; f) $90 \%$; g) $90 \%$ after the convergence criteria Final Topology.

The Fig. 5 shows the sensitivity numbers distribution in the design domain during the removing materials process. The sensitivity numbers is calculated taking account the two output points, located in the right fluid domain, and indicates the area where material should be removed for the frequency response minimization.

It must be pointed out that there are no similar examples made for FSI problems with BESO method and the obtained result shows that this methodology can be effectively applied to this kind of problems, once the pattern topology found is very similar to that presented by 


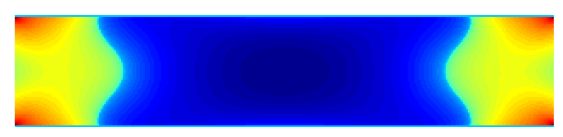

a)

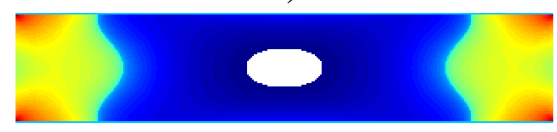

c)

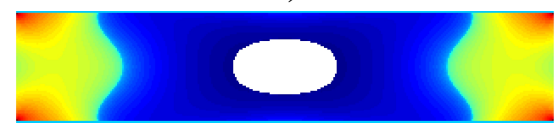

e)

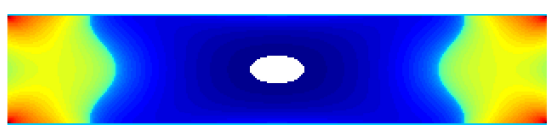

b)

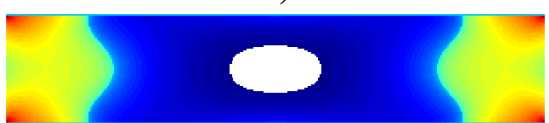

d)

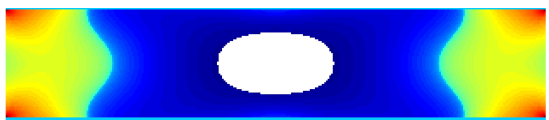

f)

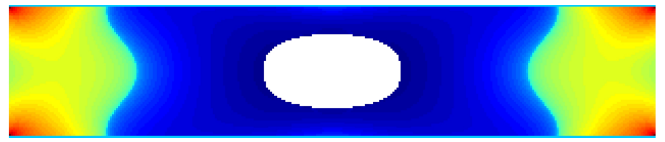

g)

Figure 5. Objective Function for Example 1 with material volume of: a) 100\% - Design Domain; b) $98 \%$; c) $96 \%$; d) $94 \%$; e) $92 \%$; f) $90 \%$; g) $90 \%$ after the convergence criteria Final Topology.

Xie and Steven [18] for a cantilever beam, in which the boundary conditions are similar to this case, which confirms the physical meaning of the obtained topology.

\subsection{Example 2}

The system of the second example is shown in Fig. 6. The materials properties are the same used in the first example. All the parameters for the BESO method is the same for the first example except the final volume which is different for each frequency of excitation. The mesh in the design domain is divided in $32 \times 320$ four-node quadrilateral elements.

This example seeks to minimize the pressure in one output point located in the right fluid domain for six excitation frequencies in the left fluid domain. The incoming wave amplitude of the excitation on the input point is $1 \mathrm{~Pa}$.

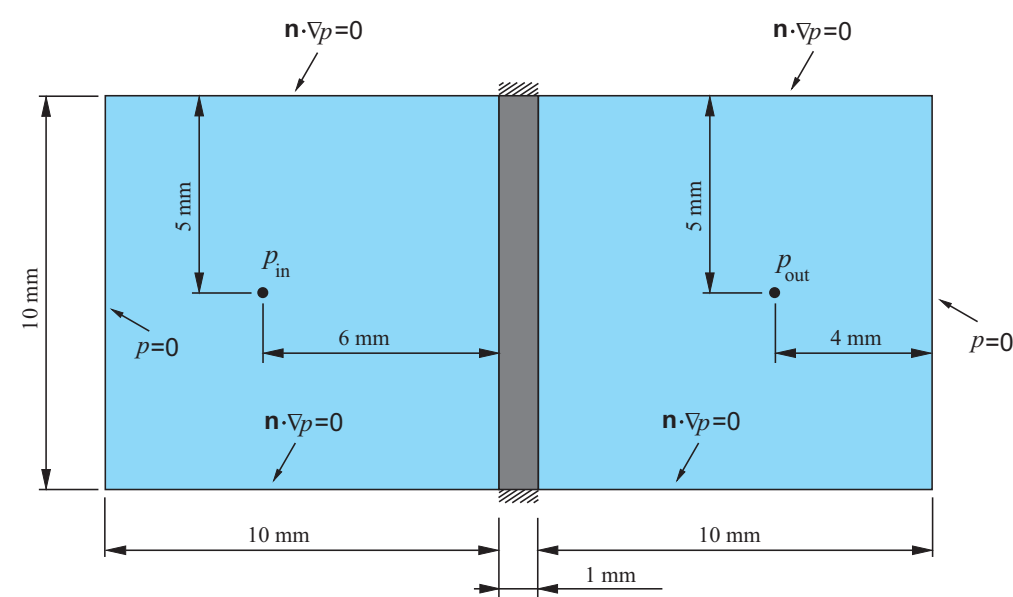

Figure 6. Model considered for optimization. 
Based on the frequency response for this system six excitation frequencies was chosen. The first three frequencies are close of the first three natural frequencies of the system, in the left side of the natural frequencies in the FRF curves, Fig. 7. The second group of excitation frequencies is chosen to be in the right side of the natural frequencies, Fig. 8. This division was done based on the different consequences of the optimization process for the FRF curves of each group, as explained in Section 3. In the first group the excitation frequencies are on the left side of the natural frequencies and in the second group they are on the right side of the natural frequencies.

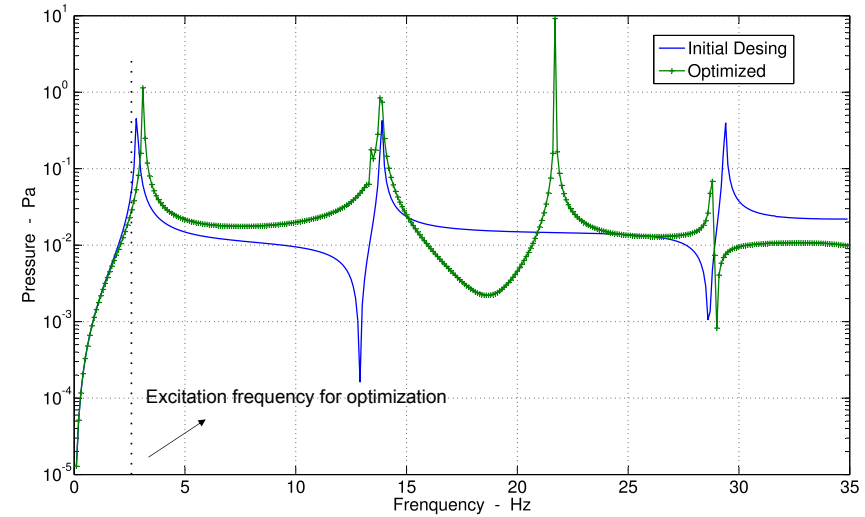

a)

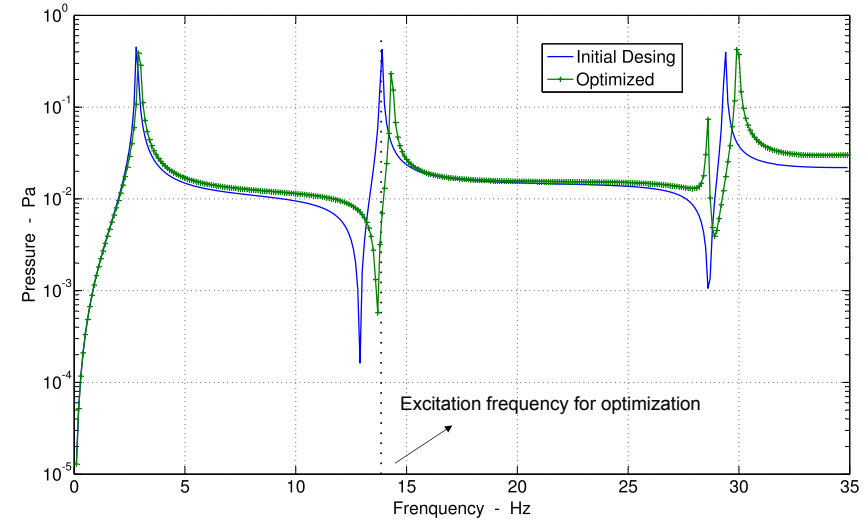

b)

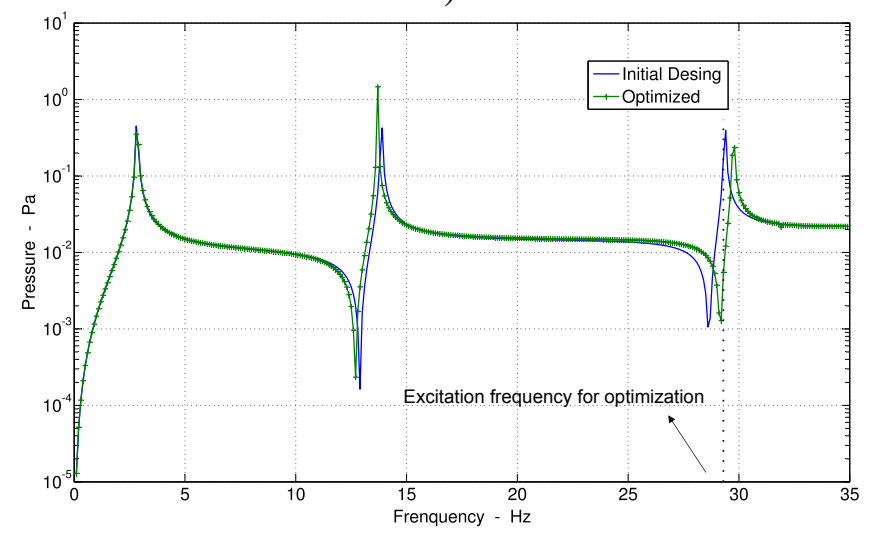

c)

Figure 7. FRF for first group of excitation frequencies.

Initial and final frequency responses for pressure in the six cases are shown in Figs. 


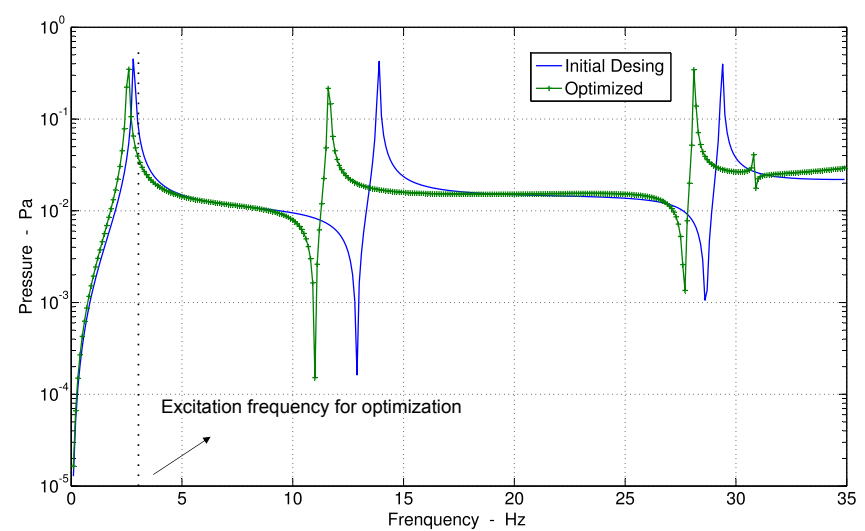

a)

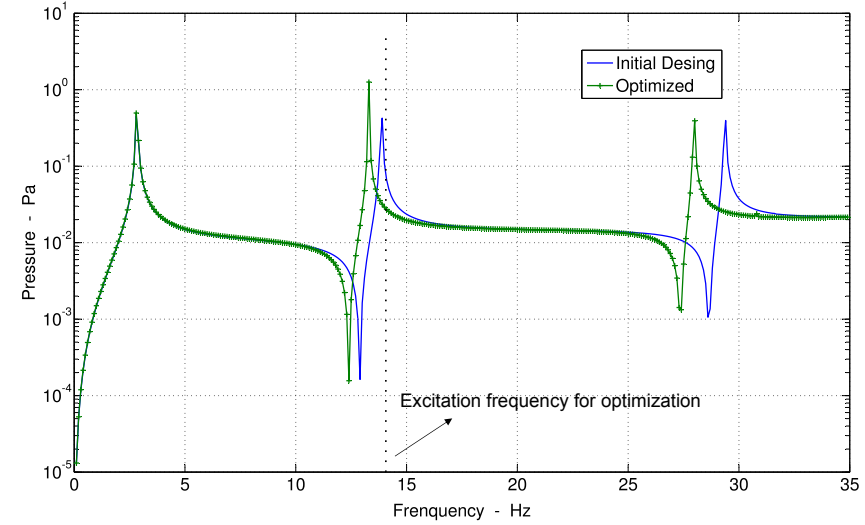

b)

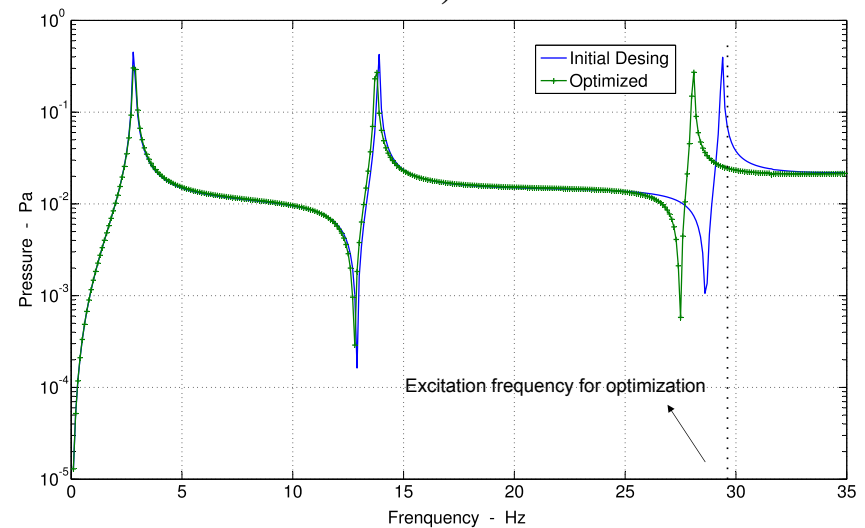

c)

Figure 8. FRF for second group of excitation frequencies.

7 and 8. It can be observed in Fig. 7 that in order to promote the minimization of the pressure the optimization process results in an increase of the natural frequencies closer of the excitation frequencies, for this first group. However, Fig. 8 shows the decrease of the natural frequencies near of the excitation frequencies, for these specific cases. It can be concluded that the optimization process can increase or decrease the natural frequencies of the system for the mean pressure minimization. It will depend of the relative position between the excitation frequency and natural frequencies in the FRF curve. The optimization process will always try to make the response to go down in the FRF curve.

Figures 9 a) c) e) presents the pressure distribution for the initial conditions and the 
shape of vibration for the three different frequencies and figures 9 b) d) f) present the final topology obtained for each case and the final pressure distribution after the optimization process for the first group of frequencies.

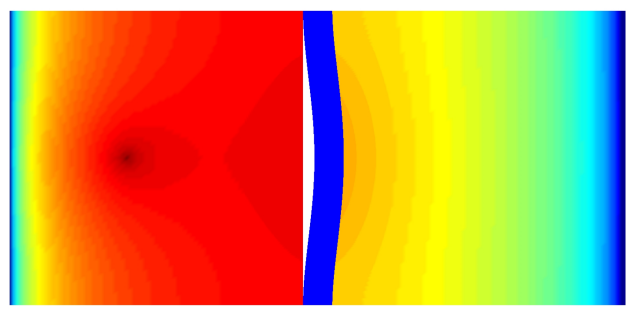

a)

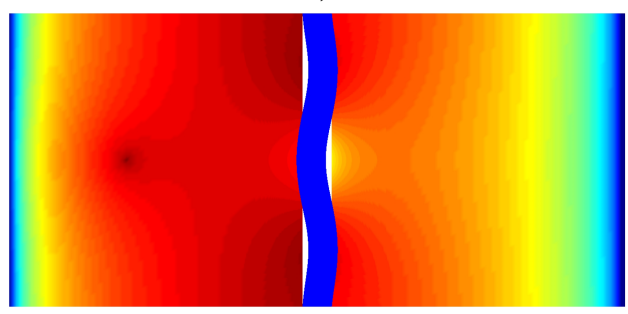

c)

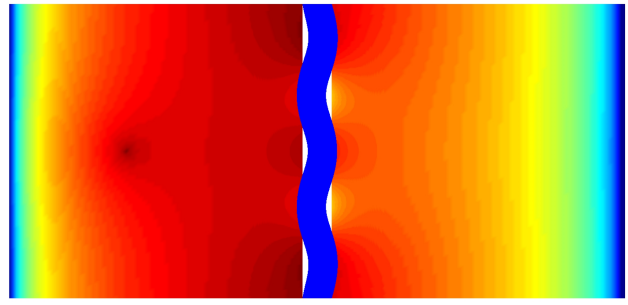

e)

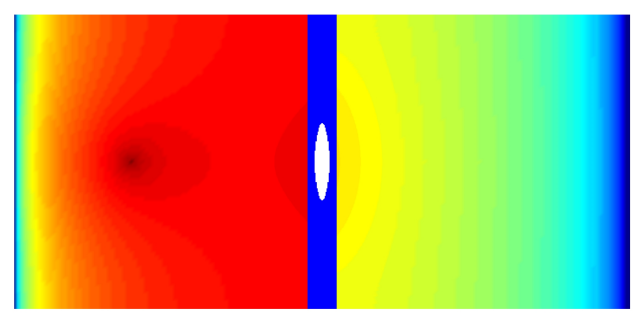

b)

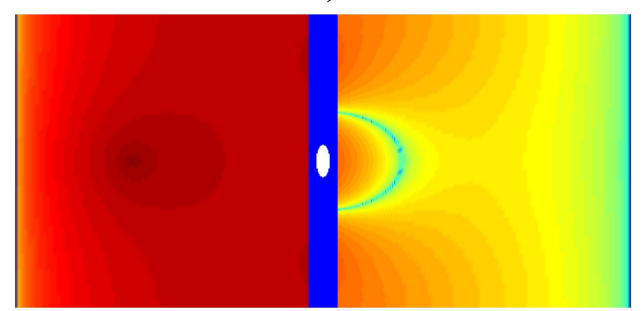

d)

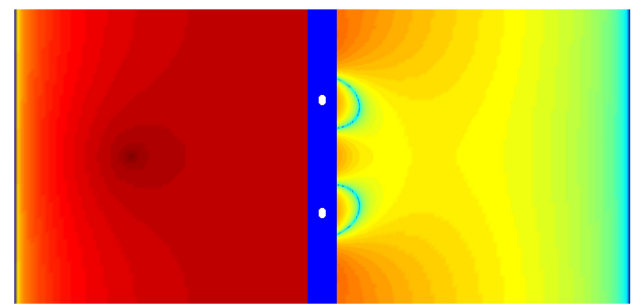

f)

Figure 9. First set of topologies for different excitation frequencies: a) $2.6 \mathrm{~Hz}-100 \%$; b) 2.6 $\mathrm{Hz}-90 \%$; c) $13.8 \mathrm{~Hz}-100 \%$; d) $13.8 \mathrm{~Hz}-96 \%$; e) $29.3 \mathrm{~Hz}-100 \%$; f) $29.3 \mathrm{~Hz}-98 \%$;

For the second group of frequencies the figures 10 a) c) e) show the pressure distribution in the initial conditions for the three different frequencies and figures $10 \mathrm{~b}$ ) d) e) show the final topology obtained for each case and the final pressure distribution after the optimization process.

In each group of frequencies it can be noted that for lower frequencies, it is necessary to remove a greater volume of material than for higher frequencies in order to obtain the same reduction of the pressure in the output point. The total reductions of the mean pressure in the output point for these six cases are shown in Tab. 3 .

Table 3. Pressure reduction for different frequencies.

\begin{tabular}{|c|c|c|c|c|c|c|}
\hline & \multicolumn{3}{|c|}{$1^{\text {st }}$ case } & \multicolumn{3}{c|}{$2^{\text {nd }}$ case } \\
\hline $\begin{array}{c}\text { Excitation } \\
\text { Frequencies (Hz) }\end{array}$ & 2,6 & 13,8 & 29,3 & 2,9 & 14 & 29,6 \\
\hline Initial pressure (Pa) & $5,49 \mathrm{E}-02$ & $1,64 \mathrm{E}-01$ & $1,70 \mathrm{E}-01$ & $2,19 \mathrm{E}-01$ & $1,08 \mathrm{E}-01$ & $7,15 \mathrm{E}-02$ \\
\hline Final pressure (Pa) & $2,89 \mathrm{E}-02$ & $3,18 \mathrm{E}-03$ & $5,49 \mathrm{E}-03$ & $4,86 \mathrm{E}-02$ & $2,92 \mathrm{E}-02$ & $8,57 \mathrm{E}-03$ \\
\hline Reduction & $47,34 \%$ & $98,06 \%$ & $96,76 \%$ & $77,79 \%$ & $72,91 \%$ & $88,01 \%$ \\
\hline
\end{tabular}




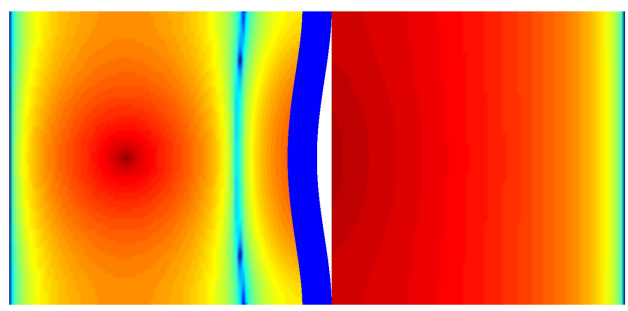

a)

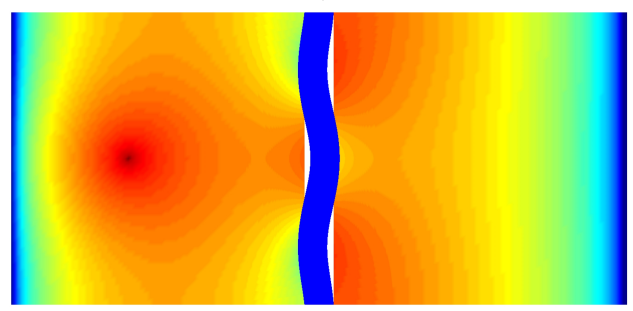

c)

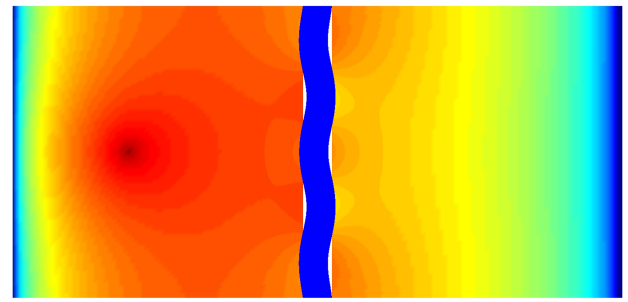

e)

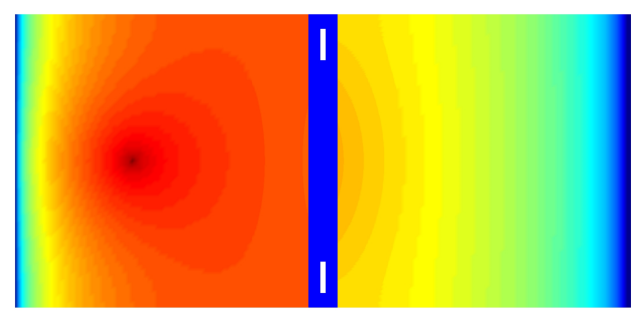

b)

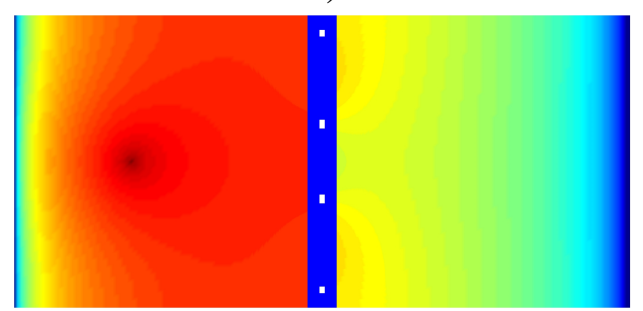

d)

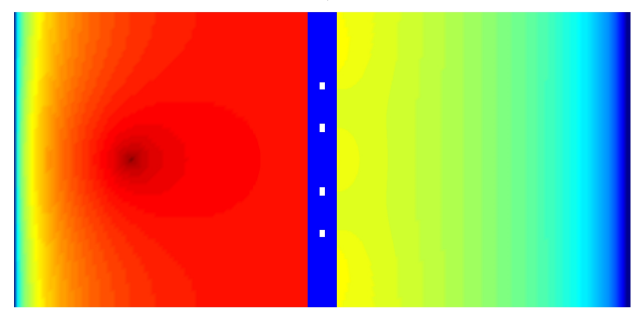

f)

Figure 10. Second set of topologies for different excitation frequencies: a) $2.9 \mathrm{~Hz}-100 \%$; b) $2.9 \mathrm{~Hz}-96 \%$; c) $14.0 \mathrm{~Hz}-100 \%$; d) $14.0 \mathrm{~Hz}-98 \%$; e) $29.6 \mathrm{~Hz}-100 \%$; f) $29.6 \mathrm{~Hz}-99 \%$;

\section{Conclusions}

In this paper we have extended the use of the BESO method to a class of fluid-structure systems under imposed pressure excitation. For this kind of problem, the sensitivity numbers were presented. The objective function was the minimization of pressure in certain points of the fluid domain. For a number of excitation frequencies the methodology presented here was capable to minimize the pressure and converge to optimized topologies. The efficiency of the method is demonstrated for a good range of frequencies. In particular for higher frequencies a great minimization of the pressure is achieved with just a small percentage of material removed. For future works, movable interfaces between the fluid and structure domain will be considered as well the extensions to three dimensions models.

\section{Acknowledgments}

The authors wish to acknowledge the financial support from $\mathrm{CNPq}$ (National Council for Scientific and Technological Development) for carrying out this work. 


\section{REFERENCES}

[1] Bendsoe, M. P. and Sigmund, O., "Topology Optimization - Theory, Methods and Applications". Berlin, Heidelberg, Springer-Verlag, 2003.

[2] Diaz, A. A. and Kikuchi, N., "Solutions to shape and topology eigenvalue optimization using a homogenization method". Int. J. Numer. Methods. Eng. 35, 1487-1502, 1992.

[3] Ma, Z. D. and Kikuchi, N. and Cheng, H. C., "Topological design for vibrating structures". Comp. Meth. App. Mech. 121, 259-280, 1995.

[4] Yoon, G. H., “Topology Optimization for stationary fluid-structure interaction problems using a new monolithic formulation”. Int J Numer Meth Eng 82, 591-616, 2010.

[5] Silva, F. I. and Pavanello, R., "Synthesis of porous-acoustic absorbing systems by an evolutionary optimization method". Eng Optimiz 42, 887-905, 2010.

[6] Sigmund, O. and Clausen, P. M., "Topology optimization using a mixed formulation: An alternative way to solve pressure load problems". Comput Methods Appl Mech Eng 196, 1874-1889, 2007.

[7] Xie, Y. M., Steven, G. P., "A simple evolutionary procedure for structural optimization". Comput Struct 49, 885-896, 1993.

[8] Querin, O. M. and Steven, G. P., "Evolutionary structural optimisation (ESO) using a bidirectional algorithm”. Eng Comput 15, 1031-1048, 1998.

[9] Huang, X. and Xie, Y. M, "Convergent and mesh-independent solutions for the bidirectional evolutionary structural optimization method". Finite Elem Anal Des 43, 10391049, 2007.

[10] Huang, X. and Xie, Y. M, "Evolutionary topological optimization of vibrating continuum structures for natural frequencies". Comput Struct 88, 357-364, 2010.

[11] Rozvany, G. I. N., "A critical review of established methods of structural topology optimization". Struct Multidiscip Optimiz 37, 217-237, 2009.

[12] Huang, X. and Xie, Y. M, "A further review of ESO type methods for topology optimization". Comput Struct 41, 671-683, 2010.

[13] Xie, Y. and Huang, X., "Evolutionary Topology Optimzation of Continuum Structures: Methods and Applications". West Sussex, John Wiley Sons, 1st edition, 2010.

[14] Yoon, G. H., Sondergaard, J. and Sigmund, O., "Topology optimization of acousticstructure interaction problems using a mixed finite element formulation". Int J Numer Meth Eng 70, 1049-1075, 2007.

[15] Davidsson, P., "Structure-acoustic analysis; Finite element modelling and reduction methods". Doctoral Thesis. Lund University, Lund, Sweden, 2004.

[16] Nha Chu, D. and Xie, Y. M. and Hira, A. and Steven, G. P., "Evolutionary structural optimization for problems with stiffness constraints". Finite Elem Anal Des 21, 239251, 1996.

[17] Sigmund, O. and Peterson, J., "Numerical instabilities in topology optimization: A survey on procedures dealing with checkerboards, mesh-dependencies and local minima". Struct Optimiz 16, 68-75, 1998.

[18] Xie, Y. M. and Steven, G. P., "Evolutionary structural optimization for dynamic problems”. Comp. Struct. 6, 1067-1073, 1996. 